\title{
Ultra Compact Nanoporous Platinum Coating Improves Neural Recording
}

\author{
Bae, Je Hyun
}

2021-04

Bae , J H , Kim , K, Han , D \& Chung , T D 2021 , ' Ultra Compact Nanoporous Platinum

Coating Improves Neural Recording ' , Electroanalysis , vol. 33 , no. 4 , pp. 839-844 . https://doi.org/10.1002/elan.20

http://hdl.handle.net/10138/338678

https://doi.org/10.1002/elan.202060519

acceptedVersion

Downloaded from Helda, University of Helsinki institutional repository.

This is an electronic reprint of the original article.

This reprint may differ from the original in pagination and typographic detail.

Please cite the original version. 


\section{ELECTROANALYSIS}

An International Journal Devoted to Electroanalysis, Sensors and Bioelectronic Devices

\section{Accepted Article}

Title: Ultra Compact Nanoporous Platinum Coating Improves Neural Recording

Authors: Je Hyun Bae, Kayeon Kim, Donghoon Han, and Taek Dong Chung

This manuscript has been accepted after peer review and appears as an Accepted Article online prior to editing, proofing, and formal publication of the final Version of Record (VoR). This work is currently citable by using the Digital Object Identifier (DOI) given below. The VoR will be published online in Early View as soon as possible and may be different to this Accepted Article as a result of editing. Readers should obtain the VoR from the journal website shown below when it is published to ensure accuracy of information. The authors are responsible for the content of this Accepted Article.

To be cited as: Electroanalysis 10.1002/elan.202060519

Link to VoR: https://doi.org/10.1002/elan.202060519 


\section{Ultra Compact Nanoporous Platinum Coating Improves Neural Recording}

Je Hyun Bae,${ }^{a_{\uparrow}}$ Kayeon Kim, ${ }^{b \uparrow}$ Donghoon Han,${ }^{\mathrm{c}^{*}}$ Taek Dong Chung ${ }^{\mathrm{d}, \mathrm{e}^{*}}$

${ }^{a}$ Graduate School of Analytical Science and Technology, Chungnam National University,

Daejeon 34134, Republic of Korea

${ }^{b}$ Helsinki Institute of Life Sciences, Neuroscience Center, University of Helsinki, PO Box 63, FI-00271 Helsinki, Finland

'Department of Chemistry, The Catholic University of Korea, Bucheon, Gyeonggi-do 14662,

Republic of Korea

${ }^{\mathrm{d}}$ Department of Chemistry, Seoul National University, Seoul 08826, Republic of Korea

${ }^{e}$ Electrochemistry Laboratory, Advanced Institutes of Convergence Technology, Suwon, Gyeonggi-do 16229, Republic of Korea

$\dagger$ These authors contributed equally to this work.

*Author to whom correspondence should be addressed, dhan@ catholic.ac.kr (Donghoon Han), Tel.: +82 2-2164-4331; Fax: +82 2-2164-4764 thchung@ snu.ac.kr (Taek Dong Chung), Tel.: +82 2-880-4362; Fax: +82 2-887-4354 


\begin{abstract}
Neural electrodes are key tools for achieving a successful brain-computer interface and the electrodes should be small to minimize damage to neural tissue and obtain good spatial selectivity such as single unit recording. Here we show conventional platinum/tungsten neural probes can be coated with nanoporous Pt. Thanks to nanoporous $\mathrm{Pt}$ with the extremely small and uniform pores, $\mathrm{L}_{2}$-ePt, the electrode impedance could be reduced by more than 2 orders of magnitude while the apparent area was almost the same. $\mathrm{L}_{2}$ ePt coating enhanced neuronal recording of local field potential in monkeys, leading to facilitating implanted electrical devices in the nervous system.
\end{abstract}

Keywords: neural recording, nanoporous Pt, microelectrode, neural interface, electrode impedance 
Potentiometry for neural recording is a conventional and representative methodology in neuroscience, which leads to the advent of the field of electrophysiology [1]. Advances in neuroprostheses are strongly dependent on the development of electrodes with enhanced ability to record neural signals [2]. For extracellular recording of neural networks, electrodes should be as small as possible because smaller surface-area-active sites improve the spatial resolution and selectivity of the electrode. However, in general, a smaller area leads to the increased overall electrode impedance values, and hence increases noise levels [3-4]. As a result, improving the signal-to-noise ratio (SNR) of the neural recording by reducing the electrode impedance has become an important issue [5]. Surface coatings are usually employed to solve this issue as they reduce the electrode impedance [6-9]. Recently, nanostructured materials with enlarged surface areas, such as nanoporous metals [10-13], and carbon nanotubes [14-16], and graphene [17-19] have been applied as neural recording electrodes.

As the apparent size of an electrode decreases, the porosity of the electrode surface becomes critical to effectively reduce the impedance [20]. Therefore, the nanopore size and roughness factor of the electrode surface become important, particularly for electrodes on the few micro- or submicro-meter scale. To demonstrate this, we compared $\mathrm{L}_{2}$-ePt, which means the electrodeposited nanoporous Pt films in reverse micelle $\left(\mathrm{L}_{2}\right)$ phases [21] and is close to the maximum porosity without electrical double-layer overlapping [22-24], and Pt black, which forms a dendritic structure. Although Pt black is mechanically fragile and degrades over time [25], it is a representative material that increases the sensitivity of neural probes. We tried to modify a commercial neural probe with the nanoporous Pt for in vivo neural recording (Fig. 1). L2-ePt should be a promising electrode material for neural recording due to the low electrode impedance and electrochemical stability in embryo brain [26].

The electron microscopic images of the nanoporous Pts show that surface of Pt black 
has the rough and random structure with poor uniformity, whereas that of $\mathrm{L}_{2}$-ePt has the smooth and even structure at the same scale (see Fig. S1 in Supporting Information). Fig. 2 shows a comparison of the cyclic voltammogram of the bare commercial Pt/W microelectrode (Fig. 2(A)) and the coated microelectrodes with nanoporous Pt (Figs. 2(B) and (C)) in $1 \mathrm{M} \mathrm{H}_{2} \mathrm{SO}_{4}$. Coating the microelectrode with nanoporous $\mathrm{Pt}$ shows the much larger current that means the enlarged electrode area resulting from formation of nanoporous Pt. To illustrate an important example concerning the dependence of the porosity of electrodes on the scale of a few micrometers as substrates, we chose pencil-shaped microelectrodes with a few micrometer tip end. When we look at corresponding optical images (Fig. 2(D)-2(F)), although the real surface areas of $\mathrm{L}_{2}$-ePt and Pt black are estimated to be the same because of similar hydrogen-adsorption behavior, there is only a small change in the exposed apparent dimensions before and after coating with higher porosity $\mathrm{L}_{2}-\mathrm{ePt}$. However, coating with Pt black significantly expands the exposed apparent size of the probe and results in lower spatial resolution.

To investigate the decrease in the impedance of nanoporous Pt-coated microelectrodes, the AC impedance was analyzed in the frequency domain of $1 \mathrm{~Hz}-10 \mathrm{kHz}$. Figure $3 \mathrm{~A}$ shows the impedance response of bare commercial $\mathrm{Pt} / \mathrm{W}$ and $\mathrm{L}_{2}$-ePt electrodes as a function of frequency. At all the frequencies studied, the impedance of $\mathrm{L}_{2}$-ePt electrodes was significantly lower than that of bare commercial Pt/W. The electrode impedance can be easily controlled by adjusting the amount of platinum to be plated. That is, as the amount of plating increases, the electrode impedance effectively decreases. At a typical frequency of $1 \mathrm{kHz}$, the impedance of the electrode with a diameter of a few micrometers decreased from $0.3 \mathrm{M} \Omega$ at the bare electrodes to $1 \mathrm{k} \Omega$ at $\mathrm{L}_{2}$-ePt microelectrodes with roughness factor 52 and to $0.5 \mathrm{k} \Omega$ at $\mathrm{L}_{2}$-ePt microelectrodes with roughness factor 170 .

In the absence of a faraday reaction and the presence of a non-adsorbable electrolyte, 
the electrode impedance is described as capacitance. The electrode capacitance, $C_{\mathrm{e}}$, can be obtained using the following equation:

$$
C_{e}=\left(2 \pi f Z^{\prime \prime}\right)^{-1}
$$

where $f$ is the frequency and $Z$ " is imaginary parts of impedance. Fig. 3(B) shows response of electrode capacitance of bare commercial Pt/W and $\mathrm{L}_{2}$-ePt electrodes as a function of frequency. The trend is almost the opposite of Fig. 3(A). This means the decrease in electrode impedance at nanoporous electrodes is due to the increase in electrode capacitance.

Interestingly, even if the plating amount of the electrode is increased, the apparent electrode area hardly changes, as shown in Figs. 3(C) and 3(D). $\mathrm{L}_{2}$-ePt has been proved to be an effective material that can effectively lower the electrode impedance while minimizing the change in apparent area. The electrode impedance could be reduced by more than 3 orders of magnitude while the apparent area was almost the same. We also carried out impedance analysis of $\mathrm{L}_{2}-\mathrm{ePt}$ in physiological conditions for neural recordings. Fig. 4 shows the impedance of $\mathrm{L}_{2}$-ePt electrodes was significantly lower than that of bare commercial $\mathrm{Pt} / \mathrm{W}$ at all the frequencies studied in $\mathrm{pH} 7.4$ phosphate buffered saline (PBS). At a typical frequency of $1 \mathrm{kHz}$, the impedance of the electrode with a diameter of a few micrometers decreased from 1.7 $\mathrm{M} \Omega$ at the bare electrodes to $67 \mathrm{k} \Omega$ at $\mathrm{L}_{2}$-ePt microelectrodes with roughness factor 61 and to $11 \mathrm{k} \Omega$ at $\mathrm{L}_{2}-\mathrm{ePt}$ microelectrodes with roughness factor 219 . The results in $\mathrm{pH} 7.4$ PBS were similar to those in $1 \mathrm{M} \mathrm{H}_{2} \mathrm{SO}_{4}$. The reduced electrode impedance implies that the SNR of the neural recording can be significantly enhanced by reducing the thermal noise that originates from the electrode impedance [3]. Although coating with Pt black is slightly inferior to coating with $\mathrm{L}_{2}-\mathrm{ePt}$, it can also effectively lower the electrode impedance [24]. However, Pt black is not suitable for a probe type inserted into a biosystem because it is mechanically fragile.

The in vivo recording quality of the $\mathrm{L}_{2}$-ePt coated microelectrodes as neural probes 
was first tested using a monkey. The trained monkey passively fixated on a spot displayed on a computer screen throughout the recordings. The bare control microelectrode and $\mathrm{L}_{2}-\mathrm{ePt}$ microelectrode were mounted $300 \mu \mathrm{m}$ apart in a five-channel minidrive. Both electrodes were then lowered until they penetrated the dura. Compared to the bare microelectrode, the 3 4 times lower noise level of $\mathrm{L}_{2}$-ePt in the primary visual cortex (V1) of the monkey is shown in Fig. 5(A). $\mathrm{L}_{2}$-ePt coating was stable and lowered the electrode impedance effectively in rat embryo brain [26]. Thus, it was confirmed that reduced electrode impedance can improve the noise level.

Fig. 5(B) shows traces of the local field potential (LFP) recorded from both electrodes. The $\mathrm{L}_{2}$-ePt trace has a large amplitude. LFP is one of the ways to measure extracellular currents, originates from multiple sources of ionic process in the outer cell space. Instantaneous changes in ionic composition can be detected at the electrode by potentiometry. LFP samples population of neurons that gives most informative signal for studying cortical electrical activities [27]. Since it picks up signals nearby as well as distant neuronal electrical activities, LFP is a plausible signal to be used to compare between bare and $\mathrm{L}_{2}-\mathrm{ePt}$. To see neural responses to visual cell stimulation more clearly, the following equation was applied in LFP data analysis:

$$
\text { Neural response }=\frac{1}{N} \mid \text { Neural signal }{ }_{\text {stim }}-\mu\left(\text { Neural signal }_{\text {baseline }}\right) \mid
$$

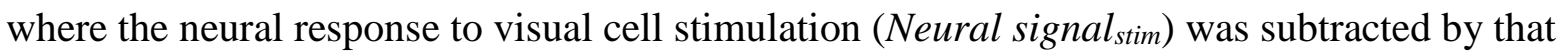
of averaged value during baseline period (-200:0ms prior to stimulation onset) to obtain the relative change of neural signal to baseline activity across $\mathrm{N}$ number of trials. We took the absolute value of the signal to make visualization more sensible from its original polarity. As shown clearly, the relative change of neural activity to visual cell stimulation to baseline activity is much larger at $\mathrm{L}_{2}$-ePt microelectrodes compared to bare control (Fig. 5(C)). Similarly, enhanced amplitudes have been previously observed for nanostructured electrodes 
$[10,14-15]$. This is because the RC time constant increases by coating the nanoporous material, so that a signal in a lower frequency can be measured. Another plausible explanation for this behavior is that the tight adhesion at the interface between the cell and the electrode due to rough surface of nanoporous structure leads to a high seal resistance related to a high SNR [13, 28-29]. In terms of the electronics and device, high electrode impedance sometimes leads to neural signal distortion [30]. Therefore, the lowered electrode impedance avoids signal distortion. Besides, the nanoporous electrode with low impedance makes it possible to detect neural signals generated further away from the electrode such as LFP coming from distant sources.

In summary, we demonstrated that nanoporous Pt with the extremely small (1-2 nm) and uniform pores can effectively lower electrode impedance while minimizing the change in apparent area and this is not possible with typical platinum black. As a result, we showed ultra compact nanoporous Pt coating improves the signal-to-noise ratio (SNR) of a neural recording in brain of living monkeys.

\section{Experimental}

Reagents. All chemicals including hydrogen hexachloroplatinate hydrate (KOJIMA chemicals, JAPAN), sodium chloride (DAE JUNG, KOREA), $t$ octylphenoxypolyethoxyethanol (Triton ${ }^{\circledR} \mathrm{X}-100$, Sigma), sulfuric acid (Sigma), and pH 7.4 phosphate buffered saline (PBS) from Sigma were used without further purification. All the aqueous solutions in this experiment were prepared with ultrapure deionized water produced by NANOpure (Barnstead).

Electrodeposition of nanoporous Pt. $\mathrm{L}_{2}$-ePt was prepared by electroplating of Pt in reverse micelle solution as described in our previous report [21,31]. Hydrogen hexachloroplatinate 
hydrate (5 wt \%), 0.3 M sodium chloride (45 wt \%), and Triton X-100 (50 wt \%) were mixed and heated to $60{ }^{\circ} \mathrm{C}$. The mixture as made was transparent and homogeneous. The temperature of the mixture solution was maintained around $40{ }^{\circ} \mathrm{C}$ using a heating bath circulator. $\mathrm{L}_{2}$-ePt was electrochemically deposited on quartz-insulated platinum/tungsten microelectrode ( $d=25 \mu \mathrm{m}, 2-3 \mathrm{M} \Omega$, ESI2ec, Thomas Recording GmbH, Germany) at $-0.1 \mathrm{~V}$ versus $\mathrm{Ag} / \mathrm{AgCl}$ to reduce formation of cracks [31]. The resulting $\mathrm{L}_{2}-\mathrm{ePt}$ electrode was soaked in deionized water to extract the Triton X-100 and it was immersed again in a new deionized water. these processes were repeated 5 times for 1 day.

The Pt black electrode was also fabricated by the electrodeposition of platinum ions from a $50 \%$ hydrogen hexachloroplatinate hydrate aqueous solution by applying a constant potential of $-0.12 \mathrm{~V}$ vs. $\mathrm{Ag} / \mathrm{AgCl}$ by the reported method [32], which is a lead-free method so that we can neglect the effects of the electrode material.

The electrochemical cleaning of the fabricated electrodes was conducted by cyclic voltammetry in a $1 \mathrm{M}$ sulfuric acid solution. The surface roughness factor of the $\mathrm{L}_{2}-\mathrm{ePt}$ was determined by measuring the area under the hydrogen adsorption peak of the cyclic voltammogram (scan rate of $0.2 \mathrm{~V} \mathrm{~s}^{-1}$ ) in $1 \mathrm{M}$ sulfuric acid, using a conversion factor of 210 $\mu \mathrm{C} \mathrm{cm}-2[33]$.

Optical images of bare and nanoporous Pt coated microelectrodes were obtained using Olympus IX71 (Olympus, Japan) equipped with DXM-1200C CCD camera (Nikon, Japan). Characterization of scanning electron microscopy (SEM) was performed using a SUPRA 55 VP Field Emission SEM (Carl Zeiss) with an acceleration voltage of $2 \mathrm{kV}$. Analysis of transmission electron microscopy (TEM) was performed using Tecnai F20 (FEI).

Electrochemical measurements. All electrochemical measurements were performed in a three electrode system by using model CHI660 (CH Instruments) as an electrochemical analyzer. 
$\mathrm{Ag} / \mathrm{AgCl}$ (3 M NaCl, MF-2052, BAS Inc.) or $\mathrm{Hg} / \mathrm{Hg}_{2} \mathrm{SO}_{4}$ (saturated $\mathrm{K}_{2} \mathrm{SO}_{4}$, RE-2C, BAS Inc.) and Pt wire (dia. $0.5 \mathrm{~mm}$, Sigma) were used as reference electrode and counter electrode, respectively. For electrochemical impedance spectroscopy (EIS), a programmed AC input with $10 \mathrm{mV}$ amplitude over a frequency range from $1 \mathrm{~Hz}$ to $10 \mathrm{kHz}$ was superimposed on the dc potential where no faradaic reaction occurs. All experiments were carried out at room temperature.

Neural recording. Extracellular potentials were recorded from the awake monkey primary visual cortex (V1) using bare and nanoporous Pt coated microelectrodes as described previously [34-35]. During the recording session, the electrode and guide tube assembly was advanced until it touched the dura, and then the electrode was further advanced by a fivechannel minidrive (Thomas Recording, Germany), penetrating the dura. Prior to recording session, the dura was thinned to prevent tissue drag during electrode penetration. The signal ground was the guide tube touching the dura. During the recording session, the animal was seated on a monkey chair with its head restrained. Neural signals from the electrode were amplified by a preamplifier with a gain of 20 (Thomas Recording, Germany), and bifurcated to the main amplifiers for LFP signals. The LFP signals were amplified with bandwidths of 0.1-140 Hz, respectively (Thomas Recording, Germany), digitized at a rate of $25 \mathrm{kHz}$ with a 16-bit resolution (PCI-6052E, National Instruments), and stored for further analysis. To obtain the neural response to visual stimulation, single cell isolation was done on-line basis. Once a single neuron was isolated, we estimated its receptive field (RF) position with visual stimuli by a hand-operated computer mouse while the monkey was fixating on a central spot of a screen. We then quantitatively determined the optimal Gabor stimulus and estimated the preferred orientation, position and the size of the stimulus that evoked the maximal neuronal activity while monkey was simply fixating at a spot [35]. Then, by presenting this optimal 
Gabor stimulus to the RF, LFP $(<140 \mathrm{~Hz})$ (Figs. 5(B) and 5(C)) were obtained.

An adult male monkey was used in this experiment. The experimental procedures were approved by the Seoul National University Institutional Animal Care and Use Committee (Permit Number: SNU-140408-7-1), and were in compliance with the U.S. National Institutes of Health guidelines. Incorporated ethical standards include an environmental enrichment program, such as routine contacts with other animals and expanded cage.

\section{ORCID}

Je Hyun Bae: 0000-0001-8840-2706

Kayeon Kim: 0000-0002-4565-9429

Donghoon Han: 0000-0003-1870-3006

Taek Dong Chung: 0000-0003-1092-8550

\section{Conflict of Interest}

The authors declare no conflict of interest.

\section{Acknowledgement}

This work was supported by research fund of Chungnam National University. We thank Prof. Choongkil Lee (Department of Psychology, Seoul National University) for help with monkey experiments.

\section{Data Availability Statement}

The data that support the finding of this study are available from the corresponding author upon reasonable request. 


\section{List of Figures}

Fig. 1. Schematic representation of the experimental setup for neural recording with a nanoporous coated probe.

Fig. 2. Cyclic voltammograms in $1 \mathrm{M} \mathrm{H}_{2} \mathrm{SO}_{4}$ at $200 \mathrm{mV} / \mathrm{s}(\mathrm{A}, \mathrm{B}$, and $\mathrm{C}$ ) and optical images showing their pencil-shaped microelectrodes (D, E, and F) before (A and D) and after modification with $\mathrm{L}_{2}-\mathrm{ePt}(\mathrm{B}$ and $\mathrm{E})$ and $\mathrm{Pt}$ black $(\mathrm{C}$ and $\mathrm{F})$. The roughness factors of $\mathrm{L}_{2}-\mathrm{ePt}$ and Pt black are 156 and 134, respectively.

Fig. 3. Total impedance (A) and electrode capacitance (B) as a function of frequency at the bare microelectrode (black square symbols) and $\mathrm{L}_{2}-\mathrm{ePt}$ microelectrodes with roughness factor 52 (red circle symbols) and 170 (blue triangle symbols) in $1 \mathrm{M} \mathrm{H}_{2} \mathrm{SO}_{4}$. The dc offset potential was $-0.25 \mathrm{~V}$ vs. $\mathrm{Hg} / \mathrm{Hg}_{2} \mathrm{SO}_{4}$. Optical images of $\mathrm{L}_{2}$-ePt microelectrodes with the roughness factors $52(\mathrm{C})$ and $170(\mathrm{D})$.

Fig. 4. Total impedance as a function of frequency at the bare microelectrode (black square symbols) and $\mathrm{L}_{2}-\mathrm{ePt}$ microelectrodes with roughness factor 61 (red circle symbols) and 219 (blue triangle symbols) in pH 7.4 PBS. The dc offset potential was $-0.2 \mathrm{~V}$ vs. $\mathrm{Ag} / \mathrm{AgCl}$.

Fig. 5. Neural recordings in the primary visual cortex (V1) of the monkey brain with the bare microelectrode (black line) and $\mathrm{L}_{2}$-ePt microelectrode (red line). (A) Noise level at resting states. (B) Local field potential traces in the range of $0.4 \sim 140 \mathrm{~Hz}$ after stimulation. (C) Relative change of LFP after stimulation to baseline activity averaged across the number of trials tested. 


\section{Figures}
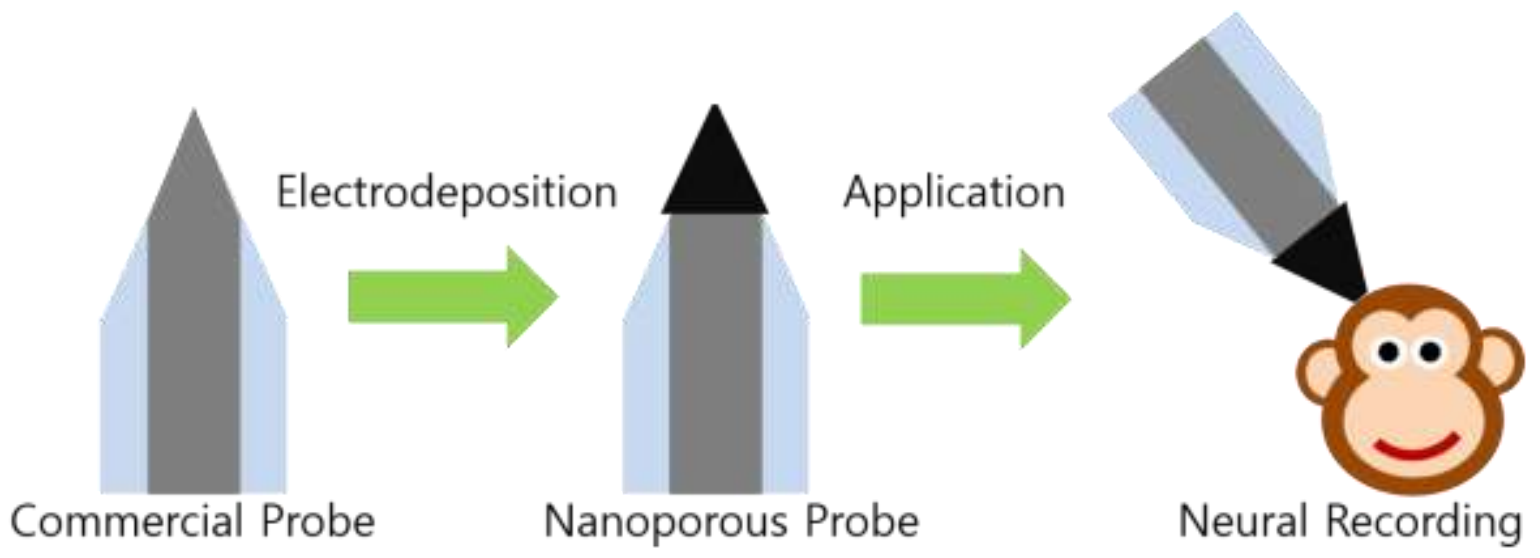

Fig. 1 

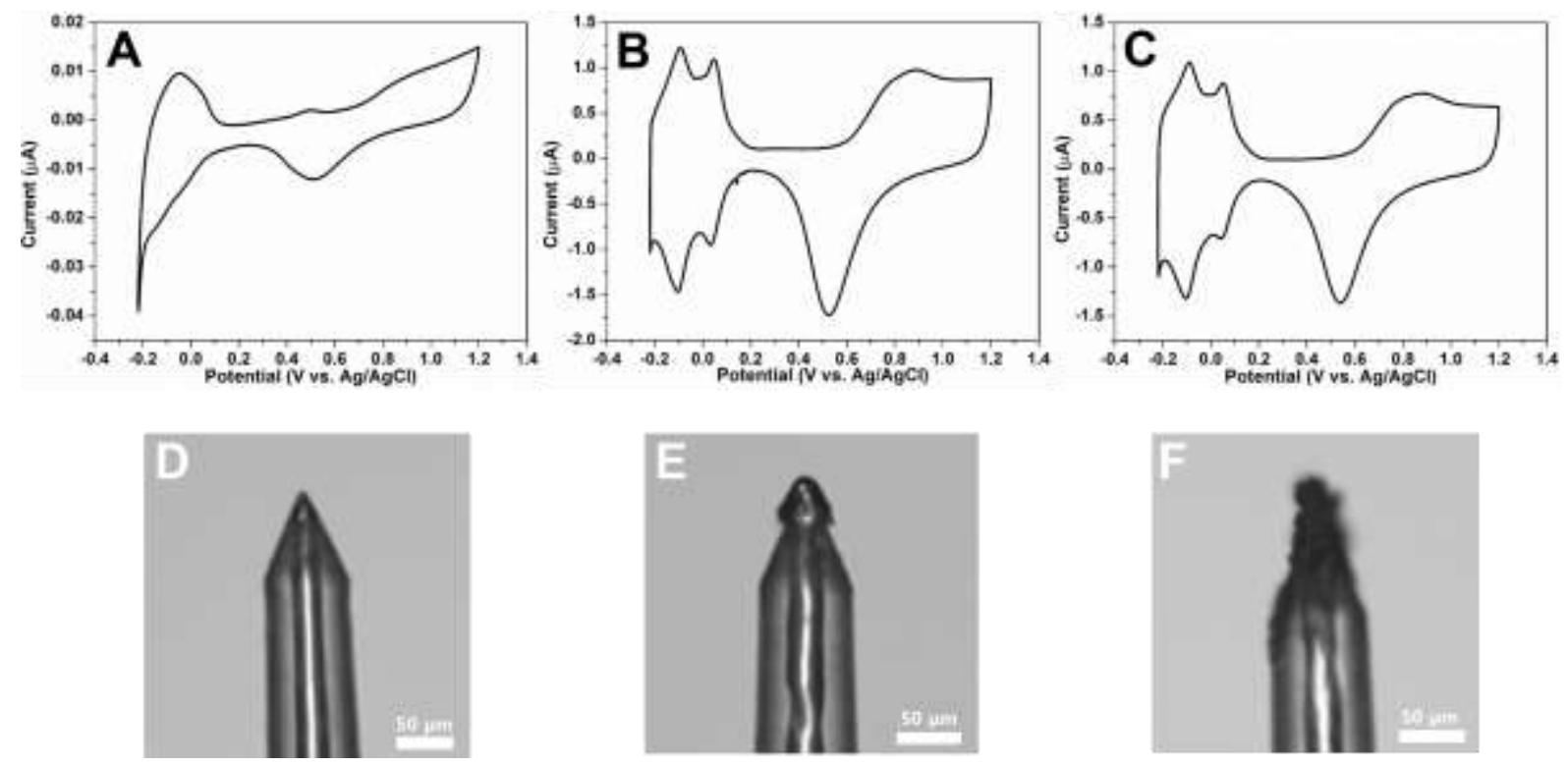

Fig. 2 

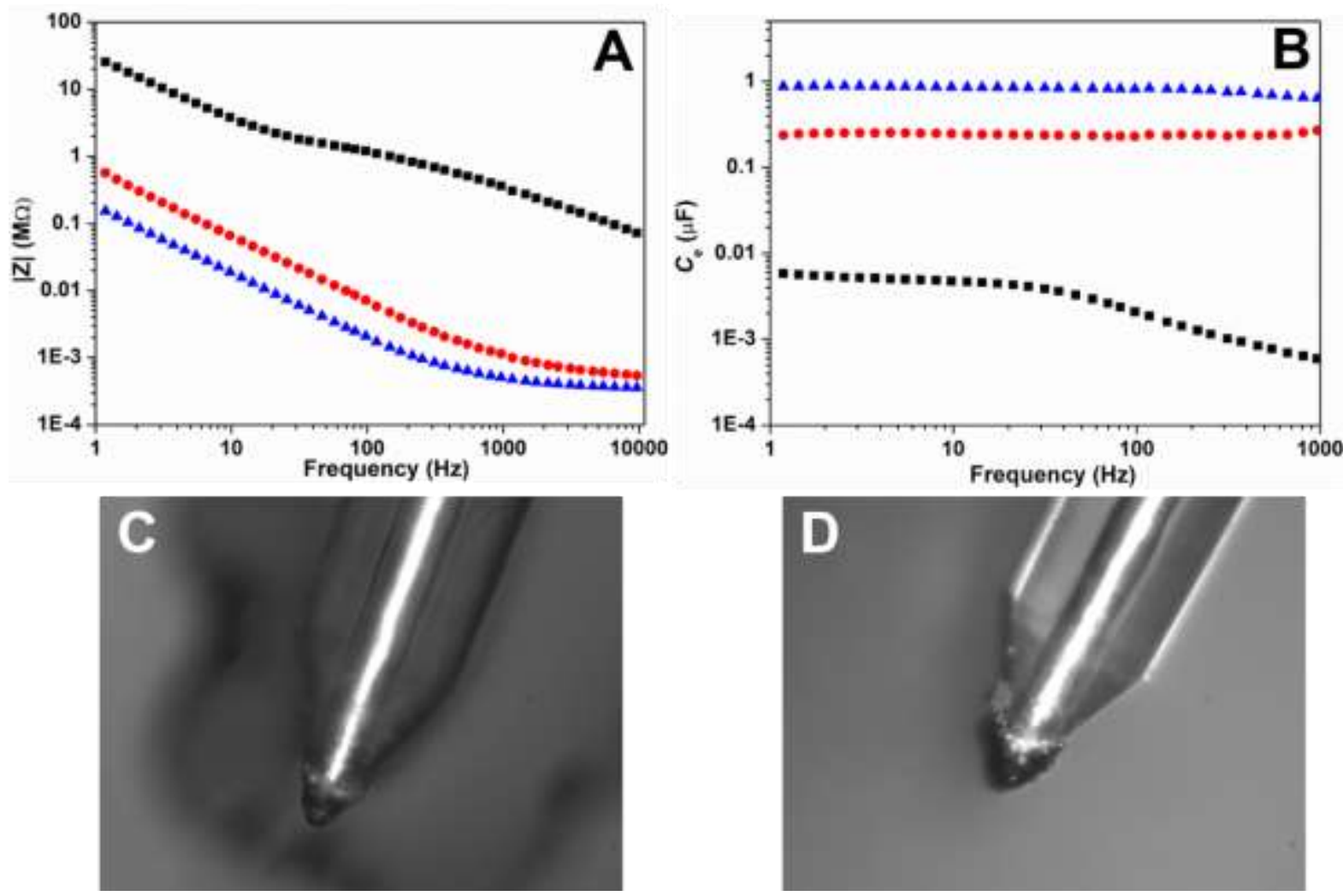

Fig. 3 


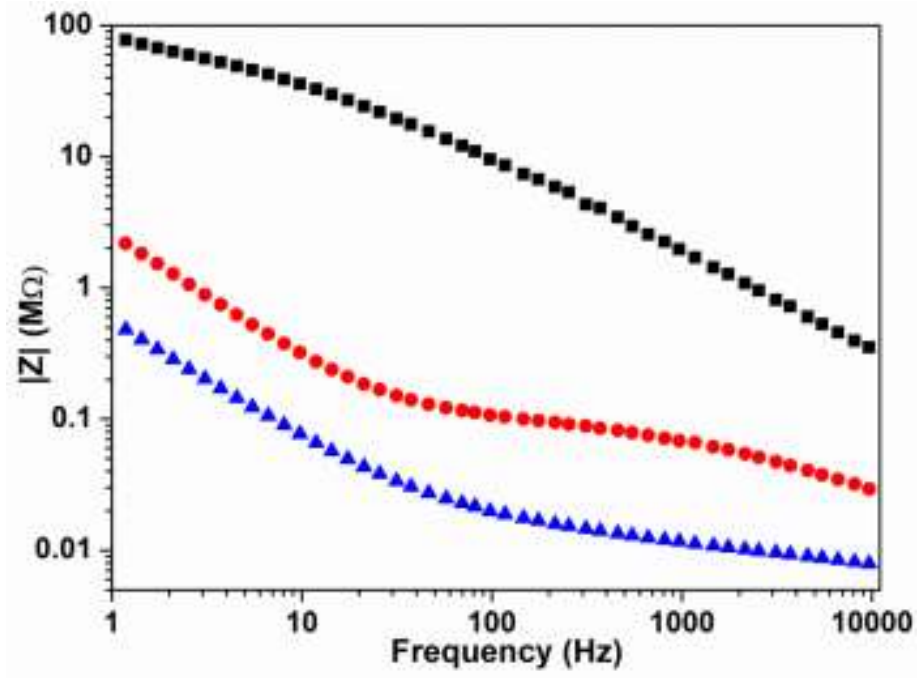

Fig. 4 

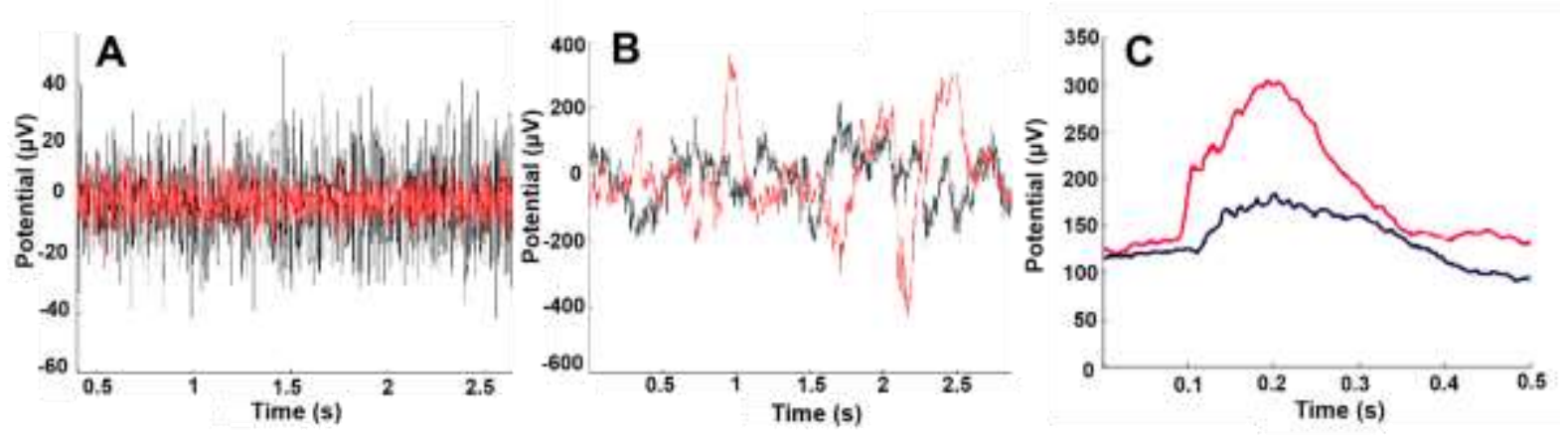

Fig. 5 
TOC
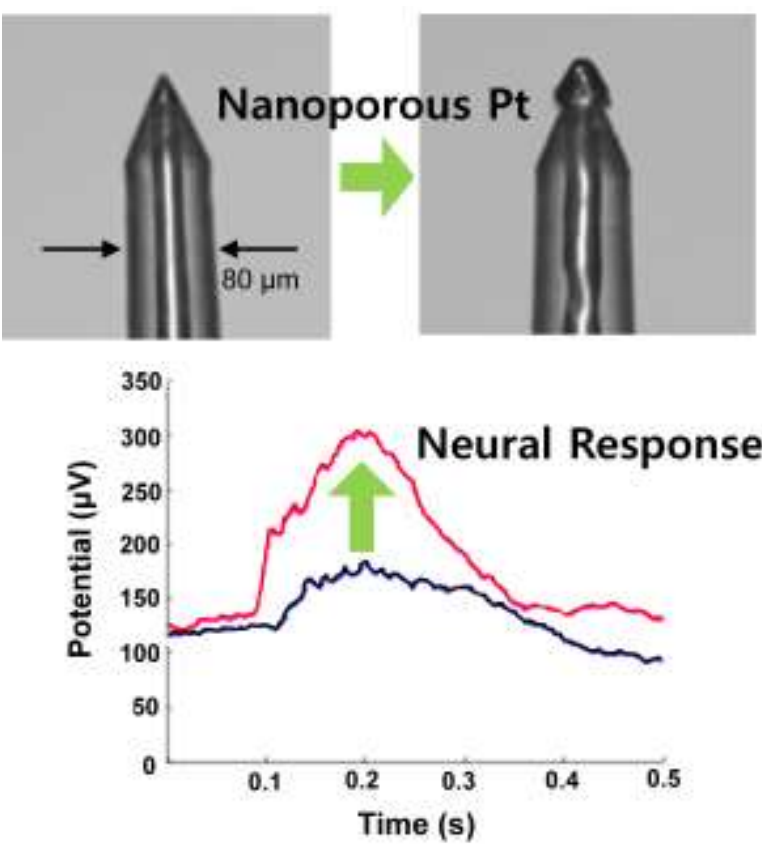


\section{References}

[1] D. Johnston, S. M.-S. Wu, Foundations of Cellular Neurophysiology, MIT Press, Cambridge, massachusetts, 1995.

[2] G. S. Hong, C. M. Lieber, Nat. Rev. Neurosci. 2019, 20, 330-345.

[3] J. B. Johnson, Phys. Rev. 1928, 32, 97-109.

[4] D. A. Robinson, Proc. IEEE 1968, 56, 1065-1071.

[5] M. Heim, B. Yvert, A. Kuhn, J. Physiol.-Paris 2012, 106, 137-145.

[6] N. Chen, L. L. Tian, A. C. Patil, S. J. Peng, I. H. Yang, N. V. Thakor, S. Ramakrishna, Nano Today 2017, 14, 59-83.

[7] J. A. Fairfield, Adv. Funct. Mater. 2018, 28, 1701145.

[8] G. H. Kim, K. Kim, E. Lee, T. An, W. Choi, G. Lim, J. H. Shin, Materials 2018, 11, 1995.

[9] Y. C. Wang, H. L. Zhu, H. R. Yang, A. D. Argall, L. Luan, C. Xie, L. Guo, Nano Res. 2018, 11, 5065-5106.

[10] E. Seker, Y. Berdichevsky, M. R. Begley, M. L. Reed, K. J. Staley, M. L. Yarmush, Nanotechnology 2010, 21, 125504.

[11] M. Heim, L. Rousseau, S. Reculusa, V. Urbanova, C. Mazzocco, S. Joucla, L. Bouffier, K. Vytras, P. Bartlett, A. Kuhn, B. Yvert, J. Neurophysiol. 2012, 108, 17931803.

[12] Y. H. Kim, G. H. Kim, A. Y. Kim, Y. H. Han, M. A. Chung, S. D. Jung, J. Neural Eng. 2015, 12, 066029.

[13] C. A. R. Chapman, L. Wang, H. Chen, J. Garrison, P. J. Lein, E. Seker, Adv. Funct. Mater. 2017, 27, 1604631.

[14] T. Gabay, M. Ben-David, I. Kalifa, R. Sorkin, Z. R. Abrams, E. Ben-Jacob, Y. Hanein, Nanotechnology 2007, 18, 035201. 
[15] E. W. Keefer, B. R. Botterman, M. I. Romero, A. F. Rossi, G. W. Gross, Nat. Nanotechnol. 2008, 3, 434-439.

[16] G. H. Kim, K. Kim, H. Nam, K. Shin, W. Choi, J. H. Shin, G. Lim, Sens. Actuator BChem. 2017, 252, 152-158.

[17] D. W. Jeong, G. H. Kim, N. Y. Kim, Z. Lee, S. D. Jung, J. O. Lee, $R S C A d v$. 2017, 7, 3273-3281.

[18] K. Kostarelos, M. Vincent, C. Hebert, J. A. Garrido, Adv. Mater. 2017, 29, 1700909.

[19] K. Z. Wang, C. L. Frewin, D. Esrafilzadeh, C. C. Yu, C. Y. Wang, J. J. Pancrazio, M. Romero-Ortega, R. Jalili, G. Wallace, Adv. Mater. 2019, 31, 1805867.

[20] G. Gabriel, R. Gomez-Martinez, R. Villa, Physiol. Meas. 2008, 29, S203-S212.

[21] S. Park, S. Y. Lee, H. Boo, H.-M. Kim, K.-B. Kim, H. C. Kim, Y. J. Song, T. D. Chung, Chem. Mat. 2007, 19, 3373-3375.

[22] H. Boo, S. Park, B. Y. Ku, Y. Kim, J. H. Park, H. C. Kim, T. D. Chung, J. Am. Chem. Soc. 2004, 126, 4524-4525.

[23] J. H. Bae, J.-H. Han, T. D. Chung, Phys. Chem. Chem. Phys. 2012, 14, 448-463.

[24] J. H. Bae, C. M. Kang, H. Choi, B. J. Kim, W. Jang, S. Y. Lim, H. C. Kim, T. D. Chung, Anal. Chem. 2015, 87, 2443-2451.

[25] S. Park, Y. J. Song, H. Boo, T. D. Chung, J. Phys. Chem. C 2010, 114, 8721-8726.

[26] B. J. Kim, Y. R. Kim, M. Seo, E. J. Kim, J. Jeon, T. D. Chung, Chemelectrochem 2016, 3, 2189-2195.

[27] G. Buzsaki, C.A. Anastassiou, C. Koch, Nat. Rev. Neurosci. 2012, 13, 407-420.

[28] A. Hai, A. Dormann, J. Shappir, S. Yitzchaik, C. Bartic, G. Borghs, J. P. M. Langedijk, M. E. Spira, J. R. Soc. Interface 2009, 6, 1153-1165.

[29] A. Hai, J. Shappir, M. E. Spira, Nat. Methods 2010, 7, 200-202.

[30] M. J. Nelson, P. Pouget, E. A. Nilsen, C. D. Patten, J. D. Schall, J. Neurosci. Methods 
2008, 169, 141-157.

[31] S. Park, Y. J. Song, J.-H. Han, H. Boo, T. D. Chung, Electrochim. Acta 2010, 55, 2029-2035.

[32] A. Kicela, S. Daniele, Talanta 2006, 68, 1632-1639.

[33] S. Trasatti, O. A. Petrii, J. Electroanal. Chem. 1992, 327, 353-376.

[34] K. Kim, T. Kim, T. Yoon, C. Lee, PLoS One 2015, 10, e0144929.

[35] K. Kim, C. Lee, J. Neurophysiol. 2017, 118, 1361-1375. 